\title{
TAGUNG
}

\section{Die EU als internationaler Akteur jenseits traditioneller Außenpolitik - Auswirkungen des Vertrags von Lissabon}

\author{
Maira Kusch*
}

Es ist eines der wesentlichen Ziele des Vertrags von Lissabon, die Handlungsfähigkeit der Europäischen Union auf der internationalen Ebene zu stärken und die Effizienz ihrer Außenvertretung zu verbessern. Ein einheitliches Modell der EU-Außenvertretung hat er jedoch, trotz der Einführung des Europäischen Auswärtigen Dienstes (EAD), nicht geschaffen. In den meisten internationalen Foren sind neben der Europäischen Union auch die Mitgliedstaaten jeweils mit einem eigenen Sitz vertreten.

Momentan gilt das politische und wissenschaftliche Interesse vor allem den traditionellen Bereichen der Außen- und Sicherheitspolitik, dabei insbesondere der Position der Hohen Vertreterin der Union für Außen- und Sicherheitspolitik (im Folgenden Hohe Vertreterin genannt) und der Entwicklung des EAD. Doch da beinahe jedes Politikfeld neben einer internen auch eine externe Dimension umfasst, wird die Kohärenz und Effektivität der EU-Außenvertretung von weit mehr als nur außenpolitischen Aspekten bestimmt. Vor diesem Hintergrund bot die interdisziplinäre Tagung „The EU in International Fora“ die Möglichkeit, das Außenhandeln der Union in den Feldern Energie und Umwelt, Handel, Wirtschaft und Finanzen sowie Arbeit und Soziales zu vergleichen. Im Mittelpunkt standen die Fragen, welche Lehren aus früheren Erfahrungen der Europäischen Union in internationalen Organisationen und Verhandlungen gezogen werden können und welche Perspektiven sich für die Außenvertretung der Union in diesen Politikfeldern mit

\section{The EU in International Fora - Lessons for the Union's External Representation after Lisbon}

Workshop der Stiftung Wissenschaft und Politik (SWP) und des Arbeitskreises Europäische Integration (AEI) in Zusammenarbeit mit dem Forum Ebenhausen e.V. und dem Istituto per gli Studi di Politica Internazionale (ISPI)

Unterstützt von der

Europäischen Kommission

Berlin, 17. Juni 2010

\section{Begrüßung}

Daniela SCHWARZER, Stiftung Wissenschaft und Politik, Berlin

Antonio VILLAFRANCA, Istituto per gli Studi di Politica Internazionale, Mailand

Einführung: The European Union in international fora - a conceptual approach

Julia LIEB, Stiftung Wissenschaft und Politik, Berlin

Louise VAN SCHAIK, Clingendael Institute, Den Haag

Energy and Environment: Climate diplomacy, coordination at international conferences, external energy relations

Vorsitz: Severin FISCHER, Institut für Europäische Politik, Berlin

Sami ANDOURA, Notre Europe, Paris

Alexander SCHÖNFELDER, Auswärtiges Amt, Berlin

Louise VAN SCHAIK, Clingendael Institute, Den Haag

Antonio VILLAFRANCA, Istituto per gli Studi di Politica Internazionale, Mailand

* Maira Kusch, B.A., Studentin im Master-Studiengang Internationale Studien/Friedens- und Konfliktforschung, Johann Wolfgang Goethe-Universität, Frankfurt am Main. 
den neuen Instrumenten des Vertrags von Lissabon ergeben. ${ }^{1}$

Die Notwendigkeit einer europäischen Energieaußenpolitik

Die europäische Energiepolitik wurde bislang vorrangig mit Blick auf den gemeinsamen Binnenmarkt beziehungsweise eine europäische Umweltpolitik entwickelt, während auBenpolitische Dimensionen weitgehend vernachlässigt wurden. Die immer wieder aufflammende Erdgaskrise zwischen der Ukraine und Russland, von der auch EU-Mitgliedstaaten wiederholt betroffen waren, warf in den letzten Jahren jedoch zunehmend die Frage nach der Notwendigkeit einer europäischen Energieaußenpolitik auf. Die europäische Energiepolitik verfolgt im Wesentlichen die drei Ziele Energiesicherheit, Nachhaltigkeit und Wettbewerbsfähigkeit. Alle drei Themenfelder weisen sowohl eine interne als auch eine externe Dimension auf. Die Außenpolitik bleibt im Energiebereich jedoch fragmentiert und die Zielvorstellungen sind nach wie vor vage.

Als wesentliche Hemmnisse für die Entwicklung einer eigenständigen Energieaußenpolitik identifizierte Sami Andoura die heterogenen Kompetenzen, Interessen und Prioritäten der beteiligten Akteure. Neben den EU-Mitgliedstaaten spielten auch private Unternehmen sowie externe Energielieferanten eine zentrale Rolle. Obwohl Energiepolitik als geteilte Zuständigkeit in den Vertrag von Lissabon aufgenommen worden sei, spezifiziere Art. 194 Abs. 2 AEUV$^{2}$, dass die Mitgliedstaaten weiterhin Souveränität in Bezug auf die Wahl ihrer Energiequellen und des Energiemixes haben. Andoura erklärte, dass sowohl die Europäische Kommission als auch das Europäische Parlament in regelmäßigen Abständen eine europäische Energieaußenpo-
Trade: World Trade Organization, economic partnership agreements, bilateral dimension of Free Trade Agreements

Vorsitz: Bettina RUDLOFF, Stiftung Wissenschaft und Politik, Berlin

Rodolfo HELG, Istituto per gli Studi di Politica Internazionale, Carlo Cattaneo University, Castellanza

Stephen WOOLCOCK, London School of Economics, London

Economics and Finance: External representation of Economic and Monetary Union, International Monetary Fund, World Bank, multilateral economic fora

Vorsitz: Daniela SCHWARZER, Stiftung Wissenschaft und Politik, Berlin

Theodor MARTENS, Europäische Zentralbank, Frankfurt am Main

Steffen MEYER, Bundesministerium der Finanzen, Berlin

Francesco PASSARELLI, Istituto per gli Studi di Politica Internazionale, Bocconi University and University of Teramo

Labour and Social Policy: European Union at International Labor Organization

Vorsitz: Sarah KRIEG, Humboldt-Universität zu Berlin

Gisbert BRINKMANN, Bundesministerium für Arbeit und Soziales, Berlin

Rudi DELARUE, Büro der International Labor Organization für die EU und die Benelux-Staaten, Brüssel

Bregt SAENEN, Universität Gent

\section{Schlussfolgerungen und Diskussion}

Nicolai VON ONDARZA, Stiftung Wissenschaft und Politik, Berlin

litik sowie einen einheitlichen Auftritt der Europäischen Union fordern. Die Mitgliedstaaten bemühten sich lediglich um eine bessere Kohärenz und Koordination ihrer Positionen.

1 Die Ergebnisse der Tagung sind in einen Sammelband eingegangen: Julia Lieb/Nicolai von Ondarza/Daniela Schwarzer (Hrsg.): The European Union in International Fora - Lessons for the Union's External Representation after Lisbon, Baden-Baden im Erscheinen.

2 Vertrag über die Arbeitsweise der Europäischen Union, in: Amtsblatt der EU, Nr. C 83 vom 30. März 2010, S. 47-199. 
Die EU-Diplomatie im Energiebereich gestalte sich daher sehr schwierig, da es an einschlägigen supranationalen Kompetenzen mangele und nicht klar sei, welches EU-Organ über eine Berechtigung zum Handeln verfüge. Die externe Energiepolitik der Europäischen Union sei weiterhin Aufgabe der Mitgliedstaaten, auf europäischer Ebene werde lediglich die externe Dimension des internen Politikfeldes reflektiert.

Nach Ansicht von Alexander Schönfelder würden die Bestimmungen des Vertrags von Lissabon zunächst wenig an der Praxis der europäischen Energiepolitik ändern. Wie viele andere Mitgliedstaaten der Europäischen Union vertrete auch Deutschland die Haltung, dass Unternehmen die wesentliche Triebkraft im Rahmen einer europäischen Energieaußenpolitik bleiben sollten. Aufgrund schwindender Gasreserven in Europa bei gleichzeitig zumindest moderat steigendem Bedarf seien zusätzliche Erdgasimporte notwendig, für die wiederum die erforderliche Infrastruktur vorhanden sein müsse. Die Nabucco-Pipeline sei ein positives Beispiel dafür, wie die europäische Energieaußenpolitik in ihrer gegenwärtigen Ausprägung funktioniere. Bauvorhaben wie die Nabucco- oder Nord Stream-Pipeline würden von privaten Unternehmen initiiert, die sich nicht nur um die Finanzierung und Durchführung des Projekts kümmerten, sondern sich gleichermaßen um die Kontakte zu beteiligten Partnerländern und notwendige Vertragsabschlüsse bemühten. Seiner Meinung nach sei es fraglich, ob ein besserer Weg für die Abwicklung derartiger Projekte bestehe - beispielsweise wenn die Kommission oder ein Konsortium von Mitgliedstaaten das benötigte Erdgas direkt von den Erzeugerländern beziehen würde. Da Unternehmen normalerweise besser als nationale Regierungen wüssten, wie derartige Energievorhaben erfolgreich und profitabel umgesetzt werden können, sollte die operative Durchführung in deren Hand verbleiben. Regierungen oder die Kommission könnten die Projekte, etwa durch Gespräche mit den ausländischen Regierungen oder Unternehmen, flankierend be- gleiten. Ebenso wie Andoura verwies Schönfelder auf die nach wie vor unterschiedlichen Interessen der Mitgliedstaaten, insbesondere mit Blick auf die Ausgestaltung einer gemeinsamen Energieaußenpolitik. Die Europäische Kommission und die Mitgliedstaaten sollten sich daher zunächst darauf konzentrieren, die Rahmenbedingungen für mehr Wettbewerb im europäischen Energiemarkt zu verbessern.

Die Rolle der Europäischen Union in einer sich wandelnden Klima-Governance

Europa strebt seit geraumer Zeit eine Vorreiterrolle im internationalen Klimaschutz an. Das Scheitern des Kopenhagener Klimagipfels im Dezember 2009 hat jedoch erneut gezeigt, dass andere relevante Akteure, wie etwa die USA, keine gemeinsame Richtung mit der Europäischen Union einschlagen. Antonio Villafranca argumentierte, dass die europäische Klimaschutzstrategie in Zukunft entsprechend angepasst werden müsse. Zuallererst sei zu berücksichtigen, dass das in den letzten zwei Dekaden entwickelte mehrstufige Governance-System im Klimabereich nicht das Resultat eines bewussten und kostenwirksamen Ansatzes gewesen sei. Es seien schlicht Kompetenzen angehäuft und auf das System der Vereinten Nationen (VN) übertragen worden. Das Ergebnis sei ein unübersichtliches, mitunter ineffektives Mehrebenensystem. Im Zentrum stünden die Programme und Agenturen der VN, allen voran die VN-Klimarahmenkonvention. Die Europäische Union sei als regionaler Akteur dafür verantwortlich, die auf höchster Ebene definierten Zielvorstellungen in regionale Pläne zu übersetzen. Infolge der Unübersichtlichkeit und der zahlreichen Überschneidungen zwischen den Initiativen der einzelnen Ebenen identifizierte Villafranca die mangelnde Koordination als Hauptproblem gegenwärtiger Klimapolitik. Obgleich er den Abschluss des Kyoto-Protokolls als große Errungenschaft des bisherigen Governance-Systems anerkannte, gab er zu bedenken, dass das erwähnte Protokoll im Jahr 2012 ausläuft und darüber hinaus keine verbindlichen langfristi- 
gen Klimaschutzziele vereinbart worden sind. Die veränderten wirtschaftlichen Kräfteverhältnisse und internationalen Rahmenbedingungen dürften es zudem in Zukunft schwierig machen, eine vergleichbare Übereinkunft $\mathrm{zu}$ erreichen. Anstelle des traditionellen vertikalen Mehrebenensystems schlug Villafranca daher den Wandel zu einem horizontalen Governance-System vor, in dem die VN nach wie vor eine Rolle - insbesondere in Bezug auf das Monitoring der Emissionen - spielen könnte, allerdings nicht mehr im Zentrum aller Aktivitäten stünde. Als mögliche alternative Arena für künftige Verhandlungen nannte er die G20, da es möglicherweise leichter wäre, ein Übereinkommen zwischen lediglich 20 Staaten zu erzielen, die 85 Prozent des weltweiten Bruttoinlandsproduktes repräsentierten.

\section{Die Klimadiplomatie der Europäischen Union nach ,Lissabon ‘}

Die Außenvertretung der Europäischen Union im Klimabereich fällt Louise van Schaik zufolge in die Zuständigkeit der rotierenden Ratspräsidentschaft und der EU-Umweltminister. Auf Basis des Art. 17 EUV $^{3}$ argumentiere allerdings auch die Kommission, dass sie die Union in Klimafragen repräsentieren solle. Darüber hinaus werde diskutiert, ob die Hohe Vertreterin im genannten Bereich aktiver werden solle. Signifikant sei, dass die Bekämpfung des Klimawandels explizit in das Umweltkapitel des Vertrags von Lissabon aufgenommen und nicht der Außenpolitik zugeordnet wurde.

Van Schaik erläuterte, dass die EU-Klimadiplomatie derzeit auf der rotierenden Ratspräsidentschaft sowie einem System von Verhandlungsführern basiere, die aus verschiedenen Mitgliedstaaten ausgewählt würden, um die Union in bestimmten klimapolitischen Angelegenheiten zu repräsentieren. Die Unfähigkeit der Europäischen Union, in Kopenhagen mit einer Stimme zu sprechen, habe jedoch zunehmend die Frage aufgeworfen, ob dieses Modell effektiv ist oder aber die Verhandlungen im Gegenteil zusätzlich erschwert, da dem Ratspräsidenten die Übersicht über die Gesamtheit der Verhandlungsführer obliegt. Alternativ zum gegenwärtigen Modell wären zwei Möglichkeiten denkbar: Zum einen könnte die Kommission die gestärkten Unionskompetenzen im Klimabereich nutzen, um die Außenvertretung zu übernehmen. Einen möglichen Ansatzpunkt böten die neu geschaffene Generaldirektion Klima sowie das Amt des Kommissars für Klimaschutz. Ein anderer, diplomatischer Ansatz wäre die Außenvertretung durch die Hohe Vertreterin und den EAD. Zum jetzigen Zeitpunkt sei jedoch fraglich, ob der EAD die notwendigen Kapazitäten für eine derartige Tätigkeit haben wird. Auch der Präsident des Europäischen Rates hat sich als Akteur ins Spiel gebracht. Solange der Klimawandel ganz oben auf der politischen Agenda in Brüssel steht, stelle Letzteres nach van Schaiks Ansicht eine Option dar. Da jedoch nicht sicher sei, wie lange das Interesse an der Klimapolitik künftig Bestand habe, wäre eine der anderen beiden Varianten vorzuziehen.

\section{Die künftige Rolle der Europäischen Union im Außenhandel}

Im Außenhandel spielt die Europäische Union seit mehreren Jahrzehnten eine bedeutende Rolle. Stephen Woolcock widmete sich in seinem Beitrag daher der Frage, inwiefern die Bestimmungen des Vertrags von Lissabon die Rolle der Union im Außenhandelsbereich künftig beeinflussen werden. Der Ökonom identifizierte in diesem Zusammenhang drei zentrale Vertragsänderungen: Die gemeinsame Handelspolitik fällt fortan unter den fünften Teil des AEUV. Die Handelspolitik muss somit im Einklang mit den Grundsätzen und Zielen des auswärtigen Handelns der Union stehen. Für die Aushandlung internationaler Abkommen wurde mit Art. 218 AEUV

3 Vertrag über die Europäische Union, in: Amtsblatt der EU, Nr. C 83 vom 30. März 2010, S. 13-45. 
ein einheitliches Verfahren geschaffen, wonach es im Ermessen des Rates liegt, ob die Kommission oder aber die Hohe Vertreterin als Verhandlungsführerin auftritt. Da der Artikel jedoch unbeschadet der Bestimmungen über die ,gemeinsame Handelspolitik“4 Anwendung findet, ändere sich am bisherigen Entscheidungsverfahren im Handelsbereich de facto nichts. Der Vertrag trage aber zur Klärung offener Zuständigkeitsfragen bei, indem die ausschließliche EU-Kompetenz im Außenhandelsbereich bestätigt und der $\mathrm{Zu}$ ständigkeitsbereich der Union nunmehr auf alle wesentlichen Außenhandelsaspekte ausgedehnt wird. Fortan fielen daher alle Dienstleistungen, ausländische Direktinvestitionen sowie geistiges Eigentum in die exklusive Unionskompetenz. Klärungsbedarf herrsche derzeit noch in Bezug auf den Rechtsstatus der bilateralen Investitionsabkommen (BIT), die in der Vergangenheit von den EU-Mitgliedstaaten individuell abgeschlossen worden sind. Obwohl zum Zeitpunkt der Tagung diesbezüglich noch keine Entscheidung gefallen war, ging Woolcock davon aus, dass bereits bestehende BIT auf dem Weg des sogenannten Grandfathering-Prinzips erhalten würden.

Die größte Veränderung sah er mit Blick auf die Rolle des Europäischen Parlaments, dessen Position durch eine Reihe von Maßnahmen gestärkt worden sei. Aufgrund der Ausweitung des ordentlichen Gesetzgebungsverfahrens auf den Handelsbereich wird der Einfluss des Parlaments unter anderem in Bezug auf die Festsetzung von Anti-Dumpingoder Handelsschutzmaßnahmen erhöht. Darüber hinaus konsolidiert der Vertrag die parlamentarische Beteiligung durch den Ausschuss des Europäischen Parlaments für internationalen Handel (INTA) am Verhandlungsprozess und bekräftigt die bisher informelle Parlamentsbefugnis, über alle handelspolitischen Abkommen abstimmen zu können. Die Kommission ist zudem von nun an rechtlich verpflichtet, das Parlament bei der Aushandlung internationaler Abkommen regelmäßig über jede Entwicklung zu unterrichten. Nichtsdestotrotz gestatte der Vertrag von Lissabon dem Parlament nicht, eigenständig handelspolitische Verhandlungen zu genehmigen. Diese Kompetenz liege weiterhin beim Rat der Europäischen Union.

Zusammenfassend bleibt festzuhalten, dass die Position der Europäischen Union im Handelsbereich grundsätzlich gestärkt wurde. Im Gegensatz zu anderen Politikfeldern ist die Union in Gestalt der Kommission seit Langem als bedeutender Akteur in der Handelspolitik anerkannt. Dies wird in Zukunft vermutlich Bestand haben. Gleichzeitig wird die Europäische Union aufgrund der strukturellen Veränderungen im internationalen Handelssystem, auf die Rodolfo Helg in seinem Beitrag näher einging, und insbesondere angesichts des Wachstums Chinas und anderer aufstrebender Märkte in Zukunft aber mit einem relativen Verlust der europäischen Marktmacht rechnen müssen.

Externe Wirtschafts- und Finanzpolitik: ein gemeinsamer EU-Sitz oder verbesserte Koordinierung?

Sollte die externe Repräsentation der Europäischen Union beziehungsweise der Eurozone in internationalen Finanz- und Wirtschaftsorganisationen gestärkt werden? Und falls ja, auf welchem Weg? Im Rahmen des Panels zu Wirtschaft und Finanzen wurden hierzu unterschiedliche Antworten gegeben. Theodor Martens wies darauf hin, dass Europa auf globaler Ebene an Einfluss verloren habe und dass sich dieser Prozess in dem sich rasch wandelnden internationalen Umfeld voraussichtlich fortsetzen werde. Vor diesem Hintergrund läge es im ureigenen Interesse der Union und ihrer Mitgliedstaaten, ihr politisches und wirtschaftliches Gewicht effektiv zu bündeln und gemeinsam zu agieren. Die rechtlichen Grundlagen dazu seien seit Langem gegeben und der Vertrag von Lissabon

4 Art. 207 AEUV. 
betone in Art. 138 AEUV ausdrücklich den Gedanken einer ,einheitliche[n] Vertretung bei den internationalen Einrichtungen und Konferenzen". Hinsichtlich der praktischen Umsetzung ließen sich bestehende Vorbehalte sicherlich überwinden, wenn man die grundsätzlich positiven Aspekte einer einheitlichen Vertretung mit den Konsequenzen einer auf Dauer zersplitterten Vertretung Europas abwäge. Der Eurozone falle in diesem Prozess eine besondere Rolle zu, da nur Mitgliedstaaten stimmberechtigt seien, deren Währung der Euro sei. Derzeit stünde die Umsetzung dieses Vertragsartikels aber noch aus.

Steffen Meyer vertrat demgegenüber die Ansicht, dass es verfrüht sei, einen einheitlichen Sitz in Foren wie der G20 oder dem Internationalen Währungsfonds anzustreben, weil mehrere Staaten mit eigener Stimmberechtigung besser in der Lage seien, bestimmte Interessen durchzusetzen. Für Deutschland sei es daher wichtig, mit einem eigenen Sitz vertreten zu sein. Bereits im Europäischen Konvent sei strittig gewesen, ob die externe Vertretung der Eurozone durch einen gemeinsamen Sitz oder aber verbesserte Koordination gestärkt werden solle. Infolge dieser Uneinigkeit sei der Vertrag offen formuliert worden. Interessant sei, dass Art. 138 AEUV lediglich die Eurozone beträfe. Zudem bleibe die Rolle der Kommission unverändert. Diese könne Vorschläge unterbreiten, über die Art und Weise der externen Repräsentation entscheide aber weiterhin der Rat. Darüber hinaus gebe der Vertrag keine Antwort darauf, wie sich die einheitliche Vertretung beim Internationalen Währungsfonds oder in der G20 in den Bereichen vollziehen wird, in denen die Kompetenz weiterhin bei den Mitgliedstaaten liegt. Da es derzeit noch eine ganze Reihe derartiger Bereiche gebe, dominiere die Rolle der Mitgliedstaaten. Für die Europäische Union sei es schwierig, in diese Lücke zu stoßen. In den letzten Jahren seien allerdings relevante Fortschritte gemacht worden, insbesondere hinsichtlich der Koordination zwischen den Staaten.
Francesco Passarelli befasste sich mit der Frage: Gewinnt oder verliert die Europäische Union, wenn sie in internationalen Organisationen zusammen oder getrennt agiert - und wenn ja, in welchem Umfang? Passarelli zufolge streben Staaten in Verhandlungssituationen nach Macht und Einfluss, weil sie hoffen, etwas zu gewinnen, wenn sie in der Lage sind, die endgültige Entscheidung zu beeinflussen. Mithilfe einer spieltheoretischen Herangehensweise kam Passarelli zu dem Ergebnis, dass es für die EU-Mitgliedstaaten nicht in jedem Fall hilfreich ist, gemeinsam zu agieren. In manchen Situationen sei die qualifizierte Mehrheit so hoch, dass es besser sei, getrennt abzustimmen. Je höher der Grenzwert für die qualifizierte Mehrheit sei, desto unbedeutender sei das gemeinsame Stimmgewicht und desto weniger mache eine einheitliche EU-Position Sinn.

\section{Die europäische Arbeits- und Sozialpolitik im} Lichte des Vertrags von Lissabon

Die externe Sozialpolitik der Europäischen Union ist ein relativ junges Politikfeld. Wie Bregt Saenen erörterte, zog es die Europäische Kommission lange Zeit vor, soziale Belange mit ihrer Handelspolitik zu verknüpfen, indem sie die Einhaltung internationaler Arbeitsnormen als Bedingung für freien Handel forderte. Nachdem insbesondere die Entwicklungsländer Kritik an dieser Sozialklausel übten, wurde der Ansatz durch eine neue Strategie ersetzt. Seit 2001 setze sich die Union für eine sogenannte ,soziale Dimension der Globalisierung ' ein und konzentriere sich zunehmend auf die Zusammenarbeit mit der Internationalen Arbeitsorganisation (ILO), um ihre sozialen Ambitionen umzusetzen. Was zunächst mit einem Briefaustausch begann, hat sich im Laufe der Zeit zu einer Partnerschaft mit vielen gemeinsamen Initiativen und Ansätzen entwickelt. Dies kann jedoch nicht über eine Reihe von Schwierigkeiten in der Kooperation hinwegtäuschen: Einerseits stellen die divergierenden Mandate und Ziele die künftige Kompatibilität beider Organisationen infrage. Die Europäische Union sei eine 
marktorientierte Gemeinschaft, deren Gründungsziel die Schaffung eines Binnenmarktes gewesen sei. Die ILO bemühe sich hingegen um soziale Gerechtigkeit auf globalem Niveau. Andererseits habe sich gezeigt, dass die Europäische Union bisher nicht in der Lage war, Drittstaaten von Standards und Normen zu überzeugen, die sie selbst aktiv mitgestaltet hatte. Die normative Kraft der Union, Elemente ihrer Sozialpolitik zu exportieren, habe sich daher als begrenzt erwiesen. Ob sich an dieser Tatsache durch den Vertrag von Lissabon etwas ändern wird, bleibe abzuwarten. Obwohl der neue Reformvertrag darauf abziele, die Rolle der Union als regionalen Akteur zu stärken, sei ihre normative Kraft in der Welt weiterhin gering.

Gisbert Brinkmann vertrat die Meinung, dass der Vertrag von Lissabon zwar eine Reihe neuer Bestimmungen zur Vertretung der Europäischen Union in internationalen Organisationen festgelegt hat, diese jedoch - zumindest für die Normensetzung der ILO - im Grunde keine Neuheit darstellen. Da die Sozialpolitik eine zwischen Union und Mitgliedstaaten geteilte Kompetenz ist, unterstütze und ergänze die Europäische Union die Aktivitäten der Mitgliedstaaten entsprechend Art. 153 AEUV. Auch nach kürzlich verfasster Ansicht des Juristischen Dienstes des Rates genießen sowohl die Union als auch die Mitgliedstaaten das Recht, bei internationalen Vereinbarungen anwesend zu sein und direkt oder durch ihre Vertreter daran teilzunehmen. Im Rahmen der Beteiligung in der ILO verfüge die Union weiterhin lediglich über einen Beobachterstatus. Die Europäische Union habe zwar ein Rederecht in den verschiedenen Stadien, die zur Annahme einer Norm (Übereinkommen oder Empfehlung) führen, aber kein Stimmrecht. Aufgrund der Tatsache, dass nur Staaten Mitglieder der ILO sind, könnten nur die EU-Mitgliedstaaten Übereinkommen ratifizieren. Sie agierten dementsprechend als ,Treuhänder' für die Union innerhalb deren Zuständigkeit. Diese könne den Mitgliedstaaten die Ratifizierung nur empfehlen. Die bestehende Praxis in der ILO (die ro- tierende Präsidentschaft der Europäischen Union spricht für die EU-Mitgliedstaaten, die Kommission spricht innerhalb ihrer Zuständigkeit) trage den Interessen und rechtlichen Gegebenheiten sowohl der Mitgliedstaaten als auch der Union Rechnung und sollte, da sie sich bewährt habe, nicht geändert werden.

Rudi Delarue ergänzte, dass die geteilte Kompetenz im Bereich der Sozialpolitik nicht automatisch impliziert, dass nicht einzelne Aspekte der ILO-Konventionen in die exklusive EU-Zuständigkeit fallen. Aufgrund der Breite des Themenfeldes gebe es zahlreiche Anknüpfungspunkte an interne und externe EUPolitiken. Neben den Arbeitsministerien und sozialen Partnern würden deshalb auch Akteure aus anderen Politikfeldern, wie der Handels- und der Entwicklungspolitik, eine wichtige Rolle spielen. Ein zentraler Punkt sei, dass die Europäische Union in der Arbeitsund Sozialpolitik auch als internationaler Akteur gefragt sei: In der Vergangenheit habe der Fokus der Union zwar hauptsächlich auf Richtlinien gelegen, die den Binnenmarkt betrafen (beispielsweise im Feld des Gesundheitsschutzes oder der Arbeitnehmersicherheit). Die sich rasch wandelnden globalen Rahmenbedingungen erforderten jedoch nunmehr internationale Verhandlungen mit Staaten wie China und Indien. Ihre Bedeutung als Gesprächspartner - etwa bezüglich der Gewährleistung menschenwürdiger Arbeit nehme stetig zu. Die Europäische Union solle Delarue zufolge zwar ihre Arbeitsnormen anderen nicht aufzwingen, könne aber durchaus multilaterale Normen und Standards auf globalem Niveau verteidigen.

\section{Fazit: Die EU steht sich selbst im Weg}

Nicolai von Ondarza fasste die zentralen Erkenntnisse der Konferenz zusammen und identifizierte als auffällige Gemeinsamkeit zwischen fast allen Politikfeldern das abnehmende internationale Gewicht der Europäischen Union im Vergleich zu den USA oder aufstrebenden Mächten in Asien. Obwohl die Europäische Union in beinahe allen internati- 
onalen Foren überrepräsentiert sei und die Mitgliedstaaten in einer Form vertreten seien, die nicht ihrer realen wirtschaftlichen und politischen Stärke entspreche, führe diese Überrepräsentanz nicht dazu, dass die Union ihre
Interessen auf internationaler Ebene besser durchzusetzen vermag. Die stärkste außenpolitische Position habe die Union im Außenhandelsbereich, in welchem sie die ausschließliche Kompetenz besitzt.

\section{Europäische Schriften}

Herausgegeben vom Institut für Europäische Politik

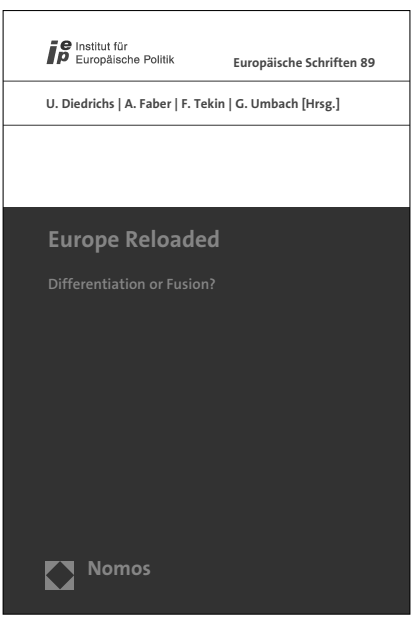

\section{Europe Reloaded}

Differentiation or Fusion?

Herausgegeben von Udo Diedrichs, Anne Faber, Funda Tekin und Gaby Umbach

2011, Band 89, 445 S., brosch., 79,- $€$ ISBN 978-3-8329-6195-4

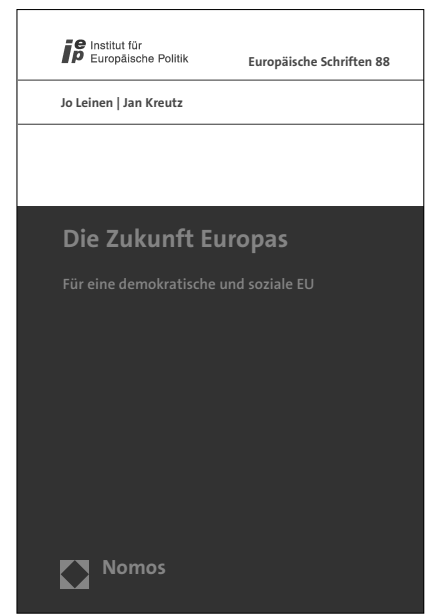

\section{Die Zukunft Europas}

Für eine demokratische und soziale EU Von Jo Leinen und Jan Kreutz 2011, Band 88, 201 S., brosch., 29,-€ ISBN 978-3-8329-6216-6

\section{Nomos}

